2011-11-17

\title{
Enhanced Quantum Dot Emission for Luminescent Solar Concentrators Using Plasmonic Interaction
}

\author{
Subhash Chandra \\ Technological University Dublin \\ Manus Kennedy \\ Technological University Dublin \\ John Doran \\ Technological University Dublin, john.doran@tudublin.ie
}

See next page for additional authors

Follow this and additional works at: https://arrow.tudublin.ie/dubenart

Part of the Optics Commons, and the Quantum Physics Commons

\section{Recommended Citation}

Chandra, S. et al (2012). Enhanced Quantum Dot Emission for Luminescent Solar Concentrators Using Plasmonic Interaction. Journal of Solar Energy Materials \& Solar Cells,vol.98, March, pp.385-390.

doi:10.1016/j.solmat.2011.11.030

This Article is brought to you for free and open access by the Dublin Energy Lab at ARROW@TU Dublin. It has been accepted for inclusion in Articles by an authorized administrator of ARROW@TU Dublin. For more information, please contact arrow.admin@tudublin.ie, aisling.coyne@tudublin.ie,gerard.connolly@tudublin.ie.

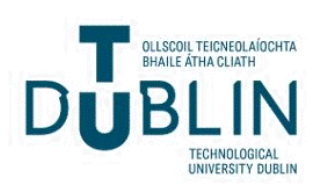


Authors

Subhash Chandra, Manus Kennedy, John Doran, Sarah McCormack, and A. J. Chatten

This article is available at ARROW@TU Dublin: https://arrow.tudublin.ie/dubenart/49 


\title{
Enhanced quantum dot emission for luminescent solar concentrators using plasmonic interaction
}

\author{
S. Chandra a,*, J. Doran ${ }^{a}$, S.J. McCormack ${ }^{b}$, M. Kennedy ${ }^{a}$, A.J. Chatten ${ }^{c}$ \\ a Dublin Energy Lab, Focas Institute, School of Physics, Dublin Institute of Technology, Camden Row, Dublin 08, Ireland \\ ${ }^{\mathrm{b}}$ School of Engineering, Trinity College Dublin, Dublin 2, Ireland \\ ${ }^{c}$ Department of Physics, Imperial College London, London SW7 2AZ, UK
}

\section{A R T I C L E I N F O}

Article history:

Received 29 July 2011

Received in revised form

14 November 2011

Accepted 17 November 2011

\section{Keywords:}

Quantum dots

Luminescent solar concentrators

Photon mode density (PMD)

Enhanced local electric field intensity

Au nanoparticles (Au NPs)

Surface plasmon resonance (SPR)

\begin{abstract}
A B S T R A C T
Plasmonic excitation enhanced fluorescence of CdSe/ZnS core-shell quantum dots (QDs) in the presence of Au nanoparticles (NPs) has been studied for application in quantum dot solar concentrator (QDSC) devices. We observe that there is an optimal concentration of $\mathrm{Au}$ NPs that gives a maximum 53\% fluorescence emission enhancement for the particular QD/Au NP composite studied. The optimal concentration depends on the coupling and spacing between neighboring QDs and Au NPs. We show the continuous transition from fluorescence enhancement to quenching, depending on $\mathrm{Au} N \mathrm{~N}$ concentration. The locally enhanced electromagnetic field induced by the surface plasmon resonance in the Au NPs leads to an increased excitation rate for the QDs. This is evidenced by excitation wavelength dependent fluorescence enhancement, where the locally enhanced field around the Au NPs is more pronounced close to the surface plasmon resonance (SPR) wavelength. However, at higher concentrations of Au NPs non-radiative energy transfer from the QDs to the Au NPs particles leads to a decrease of the emission, which is confirmed by detection of both a double exponential lifetime decay in, and a decrease in the lifetime of the QDs. The overall fluorescence emission enhancement depends on these competing effects; increased excitation rate and non-radiative energy transfer.
\end{abstract}

(c) 2011 Elsevier B.V. All rights reserved.

\section{Introduction}

Solar energy harvesting technologies are currently experiencing substantial investment and growth, driven by the aim of generating low cost (per kilowatt-hours) solar electricity. One approach is to reduce the amount of semiconductor material utilized, which is facilitated by concentrating the solar power from a large aperture area to a smaller area using inexpensive concentration techniques [1], thereby reducing the cost of the module and consequently the cost of solar electricity. The fluorescent or luminescent solar concentrator (LSC) was first suggested in late 1970s [1-7]. LSCs consist of luminescent species (organic dyes/quantum dots/rare earth materials) doped in a transparent polymer sheet. They absorb the incident solar radiation and, subsequently, re-emit luminescent light over all solid angles. The re-emitted light is guided via total internal reflection (TIR) to the sheet edges where solar cells are attached. The LSC therefore, uses active optics [6] to concentrate photons from a large aperture area to a reduced solar cell area, potentially resulting in lower cost photovoltaic devices. An additional benefit

\footnotetext{
* Corresponding author. Tel.: +35314027931.

E-mail address: subhash.chandra@mydit.ie (S. Chandra).
}

of photon concentration is an increase in photo-generated carriers and enhanced efficiency of the attached solar cell [7].

\subsection{Quantum dot solar concentrators (QDSC)}

In a quantum dot solar concentrator (QDSC) [8] the luminescent species are QDs. QDs have some potential advantages over organic dyes; i.e. the ability to tune the absorption threshold simply by the choice of dot diameter, and being crystalline semiconductors, QDs are more stable and less degradable than organic dyes [9]. QDs of various absorption thresholds and high fluorescence quantum yields (FQY) have been synthesized. For example, visible emitting core-shell (CdSe/ZnS) QDs have demonstrated fluorescence quantum yield up to $84 \%$ [10] and NIRemitting PbS quantum dots in the range of $12 \%-81 \%$ [11]. However, conversion efficiency of QDSCs developed to date [12-15] has been limited, firstly by, the low fluorescence quantum yield of the commercially available visible-emitting QDs [16] and NIR-emitting QDs $[17,18]$. Secondly, the devices suffer from re-absorption losses at higher concentrations of QDs [19-21] due to significant, or even in some cases total, overlap of the absorption and primary emission spectra. Some of these problems could be addressed by exploiting plasmonic interaction between QDs and metal nanoparticles (MNPs). The plasmonic interaction could 
be used to increase the excitation and emission rate of QDs and consequently the efficiency of the QDSC. Furthermore the reduced optimal concentration would reduce both re-absorption and module costs as QDs are currently relatively expensive at around $\sim 5960 / \mathrm{g}[22]$.

\subsection{Surface plasmon resonance (SPR)}

Metal nanostructures possess the unique optical properties of a localized surface plasmon resonance (LSPR), which is a collective oscillation of conduction band electrons, induced by excitation light as shown in Fig. 1. MNPs behave like a nanoscopic antenna [23] giving rise to strong enhancements of the local electromagnetic field intensity close to the NPs [24-26]. The enhanced electric field vector decays exponentially with distance away from the metal surface, with decay length of the order of one half of the excitation wavelength [27].When a fluorescent emitter (e.g. QDs, dye) is placed in the range of enhanced local electric field intensity, plasmonic interaction takes place, which can enhance light absorption, the excitation rate, and radiative and non-radiative decay rates of the fluorescent species.

\subsection{Plasmonic interaction}

Total fluorescence of an optical emitter is determined by radiative recombination of the excited electron-hole pair, by coupling of the excited state to the vacuum oscillations of the local environment, which competes with non-radiative processes [28-30]. This complete fluorescence process can be formulated in classical terms, where the probability of emitting a photon is related to the local electric field intensity (photonic mode density (PMD)) [31]. The fluorescence process can be written as a product of two processes: (1) Excitation by the incident field, influenced by local environments; and, (2) emission, influenced by balance of radiative and non-radiative decays. These points, above, give us an insight on how to modify the fluorescence of the optical emitter (i.e. QDs) by altering the local PMD, which can be changed using MNPs exhibiting a localized surface plasmon resonance.

There are two possible ways to enhance the fluorescence emission of a QD in the vicinity of MNPs: an increase in the excitation rate of the QDs and an increase in the fluorescence quantum yield of the QDs. The influence of the enhanced electromagnetic field on the excitation rate of the fluorescent species $\left(\Gamma_{\text {exc }}\left(r_{0}\right)\right)$, is straightforward, $\Gamma_{\text {exc }}\left(r_{0}\right)$ proportional to electric field intensity $E_{\text {exc }}\left(r_{0}, \omega\right)$ at the molecule [32]:

$\Gamma_{\text {exc }}\left(r_{0}\right) \propto\left|p E_{\text {exc }}\left(r_{0}, \omega\right)\right|^{2}$

where, $E_{\text {exc }}\left(r_{0}, \omega\right)$ is the incident plus surface plasmon enhanced electromagnetic field, $p$ the transition dipole moment, $\omega$ the transition frequency. The maximum enhancement occurs when the NP plasmon resonance wavelength coincides with the QD

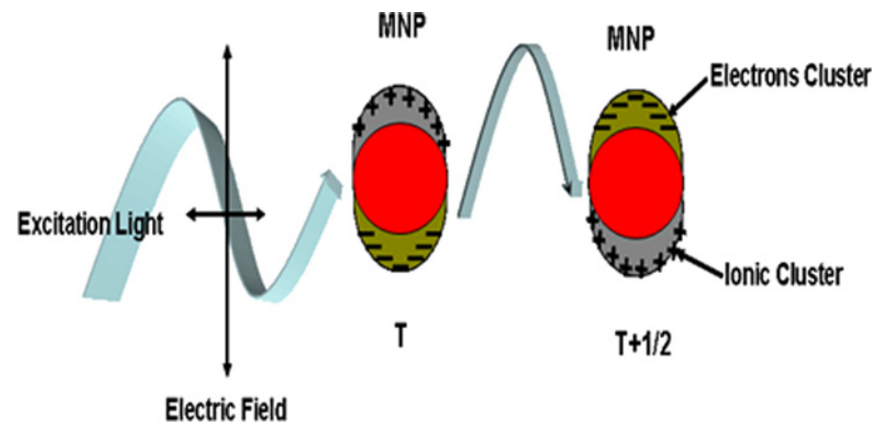

Fig. 1. Schematic representation of the surface plasmon excitation in metal nanoparticles. absorption band [33-35]. The second effect involves an increase in the fluorescence quantum yield of the optical emitter and is maximized when the MNPs resonance wavelength coincides with the QD emission band [36-38]. The total fluorescence emission rate is given by [32]

$\Gamma_{e m}=\Gamma_{e x c}\left[\frac{\gamma_{r}}{\gamma}\right]$

where, $\gamma$ is the total decay rate, $\gamma=\gamma_{n r}+\gamma_{r}$, and $\gamma_{r}$ and $\gamma_{n r}$ are the radiative and non-radiative rates, respectively.

It is clear that emission can be controlled through the modification of the local electromagnetic boundary condition (or PMD) near the optical emitter. This phenomenon depends on many parameters, such as metal type, MNP size and shape, MNP$\mathrm{QD}$ separation, and fluorescence quantum yield of the QD. The overall effect of plasmonic interaction in a given system is determined by competition between excitation enhancement (increased light absorption), emission enhancement (increased radiative decay), and quenching (increased non-radiative decay). Our work has exploited the first phenomenon.

\section{Materials and experimental detail}

\subsection{Materials}

The materials used for synthesis of the Au NPs and QD/Au NP composites were: Gold precursor gold (III) chloride trihydrate $\left(\mathrm{HAuCl}_{4} \cdot 3 \mathrm{H}_{2} \mathrm{O}\right)$ of high purity $\geq 99.9 \%$, poly vinylpyrrolidone (PVP) of $M_{\mathrm{w}} 10,000$, HPLC grade toluene, sodium hydroxide $(\mathrm{NaOH})$ of $M_{\mathrm{w}} 40.00 \mathrm{~g} / \mathrm{mol}$ (Sigma Aldrich, Ireland), and analytical grade ethanol (Lennox, Ireland). Core-shell CdSe/ZnS quantum dots (Plasma Chem, Germany) were used as the fluorescent material and clear casting epoxy resin (ABL Resin $\mathcal{E}$ Glass, UK) was used as the dispersion medium. Samples were prepared in a polymethylmethacrylate (PMMA) cuvette of $1 \mathrm{~cm}$ path length. All glassware were cleaned with detergent, acetone, and ethanol and finally rinsed with deionised water from a Millipore system.

\subsection{Characterization techniques}

A Perkin Elmer Lambda 900 UV/VIS/NIR Spectrometer was used to measure UV-vis absorption spectra. Luminescence measurements were recorded using a Perkin Elmer LS55B luminescence spectrometer, where luminescence was collected at a $90^{\circ}$ angle to the excitation beam. The fluorescence lifetime of the QD/ Au NP composites in epoxy was recorded using a time-correlated single photon counting (TCSPC) technique.

\subsection{Sample preparation}

\subsubsection{Au NPs dispersion in epoxy resin}

The PVP-stabilized spherical $(10 \pm 1 \mathrm{~nm})$ Au NPs were synthesized in ethanol by the chemical reduction of gold (III) chloride trihydrate $\left(\mathrm{HAuCl}_{4} \cdot 3 \mathrm{H}_{2} \mathrm{O}\right)$ precursor. Gold (III) chloride trihydrate $\left(\mathrm{HAuCl}_{4} \cdot 3 \mathrm{H}_{2} \mathrm{O}\right)$ was reduced in ethanol at a refluxing temperature of $80{ }^{\circ} \mathrm{C}$ in the presence of PVP $\left(M_{\mathrm{w}}=10,000\right)$ and $\mathrm{NaOH}$ [39]. These Au NPs were dispersed in epoxy resin using a magnetic stirrer as shown in Fig. 2. The epoxy resin contains two parts; resin and hardener, mixed in the ratio 100:42 by weight.

The SPR wavelength for MNPs is critically determined by four factors; the density and effective mass of the electrons, the shape of the MNPs, and the size of the charge distribution. The charge distribution depends on the local dielectric environment of the MNPs and changing this environment can affect the plasmon oscillation frequency, due to the varying ability of the surface to 


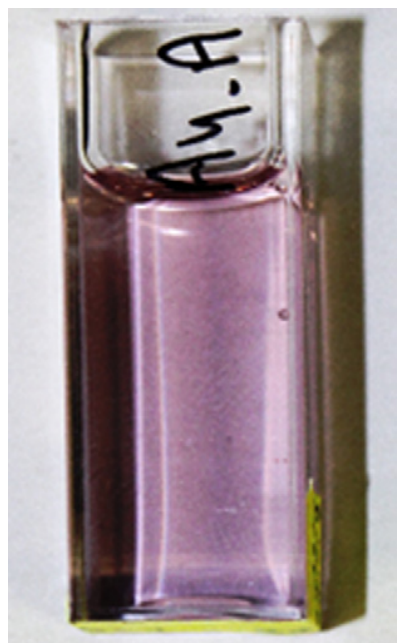

Fig. 2. Au NPs $(50.0 \mathrm{ppm})$ dispersed in epoxy and cast in a $1 \mathrm{~cm}$ path length PMMA cuvette.

accommodate the electron charge density and distribution in the MNP [40]. The SPR wavelength of Au NPs is red shifted by $13 \mathrm{~nm}$ in epoxy resin compared to that in ethanol solution, as shown in Fig. 3. This is as a result of the difference in refractive indices of ethanol and epoxy (ethanol $n \approx 1.36$ and epoxy $n \approx 1.5$ ). A change in refractive index is indicative of a change in the local dielectric environment of the Au NPs, which ultimately alters the distribution of surface electrons on the nanoparticles and consequently the SPR wavelength.

\subsubsection{Quantum dot/Au NP composite in epoxy}

This work has carried out material characterizations of QDs/Au nanoparticles composite for a new approach to luminescent solar concentrator using plasmonic interaction of Au nanoparticles. The material characterization is a very important first step to fabricate luminescent solar concentrator devices [41]. To make characterization more accurate and convenient for available characterization techniques (UV/vis Absorption Spectrometry, Luminescence Spectrometry, and Time Correlated Single Photon Counter (TCPSC)), samples were prepared in PMMA cuvette of $1 \mathrm{~cm}$ optical path length.

Core-shell type CdSe/ZnS quantum dots were used as the fluorescent material for the QDSC. Their typical absorption and emission spectra (in toluene, $0.125 \mathrm{mg} / \mathrm{ml}$ ) are shown in Fig. 4. The CdSe/ZnS QDs were dispersed in epoxy resin, which is reported as a suitable matrix material for a QDSC device, retaining the fluorescence quantum yield of QDs [41]. The CdSe/ZnS QDs/Au NPs composites were prepared as follows: first the QDs were dispersed in the hardener $(0.008 \%$, weight percentage) by magnetic stirring for $15 \mathrm{~min}$ and then sonicated for $10 \mathrm{~min}$; this was followed by the addition of the Au NPs (1.0-4.0 ppm) and stirring; then the resin was added, followed again by a further $15 \mathrm{~min}$ of stirring. Finally the mixture was cast and cured in a $1 \mathrm{~cm}$ polymethylmethacrylate (PMMA) cuvette at $25{ }^{\circ} \mathrm{C}$ for $24 \mathrm{~h}$.

\section{Results and discussion}

\subsection{Optical absorption of $Q D / A u N P$ composite}

Absorption spectra were recorded using an integrating sphere configuration in a Perkin Elmer Lambda 900 UV/VIS/NIR spectrometer. A solid epoxy sample was used as a reference during the measurements. We observed QD absorption spectra in epoxy like

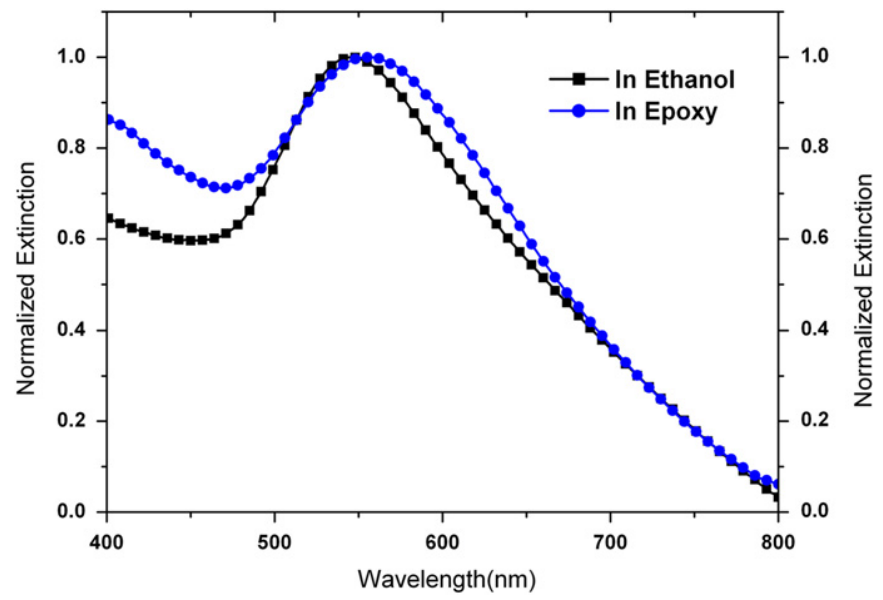

Fig. 3. Normalized extinction spectra of Au NPs (50.0 ppm) in ethanol (squares) and epoxy (solid circles). The SPR wavelength of Au NPs is red shifted in epoxy resin compared to that in ethanol.

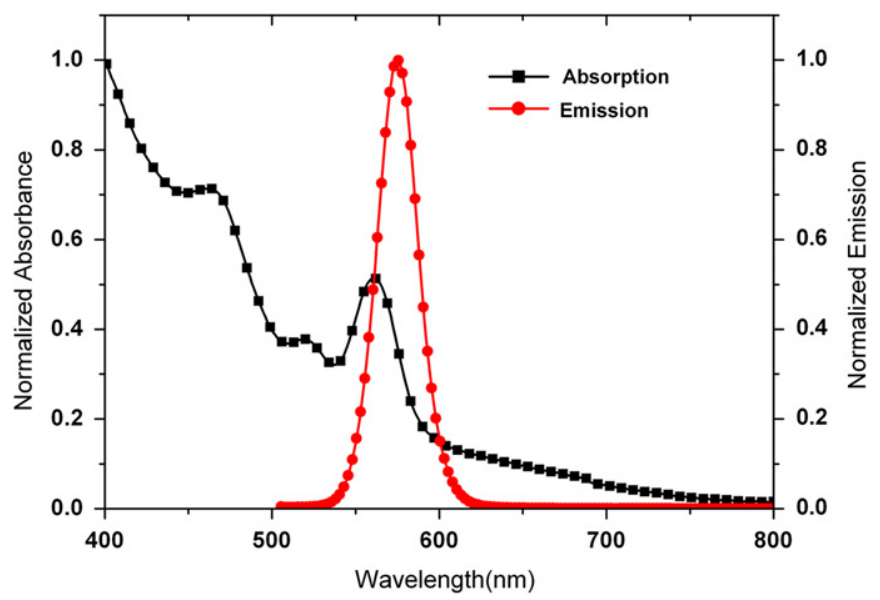

Fig. 4. Normalized absorption (squares) and emission (solid circles) spectra of the QD $570 \mathrm{CdSe} / \mathrm{ZnS}$ quantum dots in toluene $(0.125 \mathrm{mg} / \mathrm{ml})$.

that observed in toluene solution, which confirms that the QDs are uniformly dispersed in the epoxy.

Absorption increased with increasing Au NP concentration, as is shown in Fig. 5. Enhanced optical absorption can be attributed to an enhanced local photon mode density in the vicinity of the QDs due to the presence of the MNPs. The optical transition in QDs is directly proportional to the local photon mode density. In the absence of Au NPs, light is absorbed directly by the QDs. In contrast, when Au NPs are added, the incident light excites the surface plasmon (SP) modes in the Au NPs, which leads to an enhanced local photon mode density [42] (the total electric field is the sum of incident plus plasmon induced field, as discussed in Section 1.3). This can also be understood as a lengthening of the duration of the interaction time between the photon and an individual QD. This interaction time is determined by the lifetime of the surface plasmon (SP) excitation, which is typically in the range $5-10$ fs [43], and by the decay length of the SP mode from the MNPs surface.

\subsection{Fluorescence emission of $Q D / A u N P$ composite}

Fig. 6 presents the fluorescence emission spectra of QDs in epoxy with and without Au NPs. The emission peak was similar in all cases to that for the QDs in toluene; again showing that 


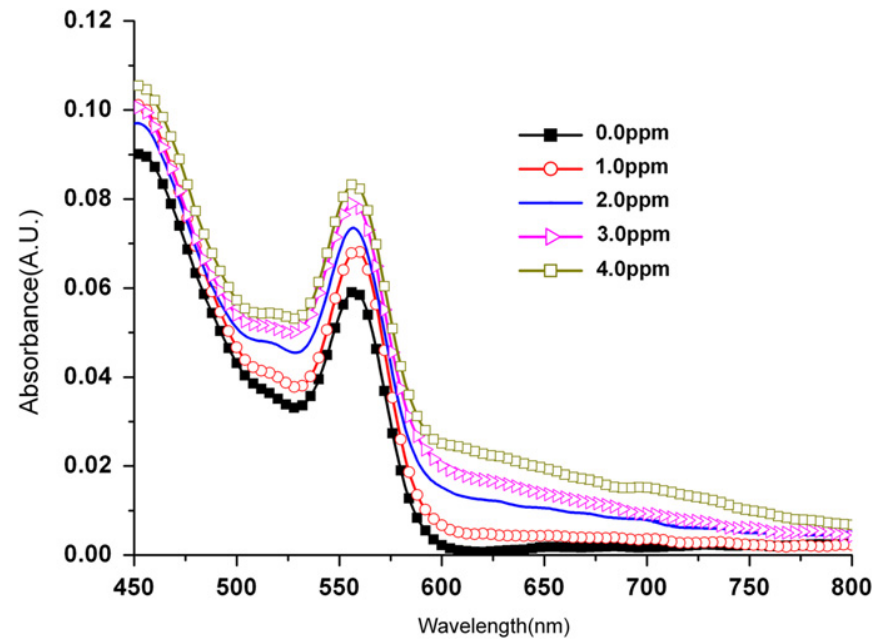

Fig. 5. UV-vis absorption spectra of the QD 570 quantum dots with varying Au NP concentration (0.0-4.0 ppm) in epoxy. (Note; the $1.5,2.5$, and $3.5 \mathrm{ppm}$ data have not been shown in this figure to keep clarity.).

uniform dispersions of QDs were achieved. We see that the fluorescence emission intensity depends sensitively on the concentration of Au NPs. As the concentration of Au NPs is increased the emission intensity is initially enhanced and, eventually, further increases of Au NP concentration leads to reduction of the emission intensity. The maximum emission intensity was observed for $2.0 \mathrm{ppm}$ of Au NPs.

The spacing between the optical emitter and MNP is another important factor that controls the plasmonic interaction and consequently the fluorescence emission. The plasmonic interaction is highly dependent on the QD-Au NP spacing, as the enhanced field decays exponentially away from the MNPs. Only emitters (i.e. QDs) located in the range of the enhanced field experience plasmonic interaction. If the QDs are too close to the MNP (less than a few nm), then the possibility exists that the excited state QD electron and hole can tunnel to the Au NP through nonradiative relaxation [34]. Therefore, an optimum spacing (controlled by MNP concentration) should exist, as has been observed in our QD/MNP composites.

The fluorescence enhancement as a function of Au NP concentration is plotted in Fig. 7. At low concentrations of Au NPs, only a small number of QDs are coupled with Au NPs via plasmonic interaction. By increasing the concentration, the proportion of QDs coupled to Au NPs increases and this leads to an increase of the total emission. However, with further increasing of Au NP concentration there is also decreased spacing between the QDs and Au NPs and the possibility of non-radiative transfer of the carriers to the NP also increases. This phenomenon leads to a decrease of the fluorescence emission intensity at concentrations of Au NPs above $2.0 \mathrm{ppm}$ in this particular QD composite, as shown in Fig. 7. These are competing effects, where increased emission due to enhanced excitation rate is offset by quenching of the emission at higher MNP concentration. These competing factors determine the overall behavior of QD/Au NP composite systems.

These results showed 53\% QD emission enhancement for this particular concentration of QDs in the presence of Au nanoparticles. This proved that QD concentration could be decreased, without compromising the total emission, using the plasmonic interaction of $\mathrm{Au}$ NPs in a luminescent solar concentrator. This would lead to a decrease of the re-absorption losses in a device, which primarily occurs due to higher concentration of luminescent species in luminescent solar concentrators [19-21]. It is reasonable to expect a change in the overall efficiency of a luminescent solar concentrator when this optimized QDs/Au NPs composite is used

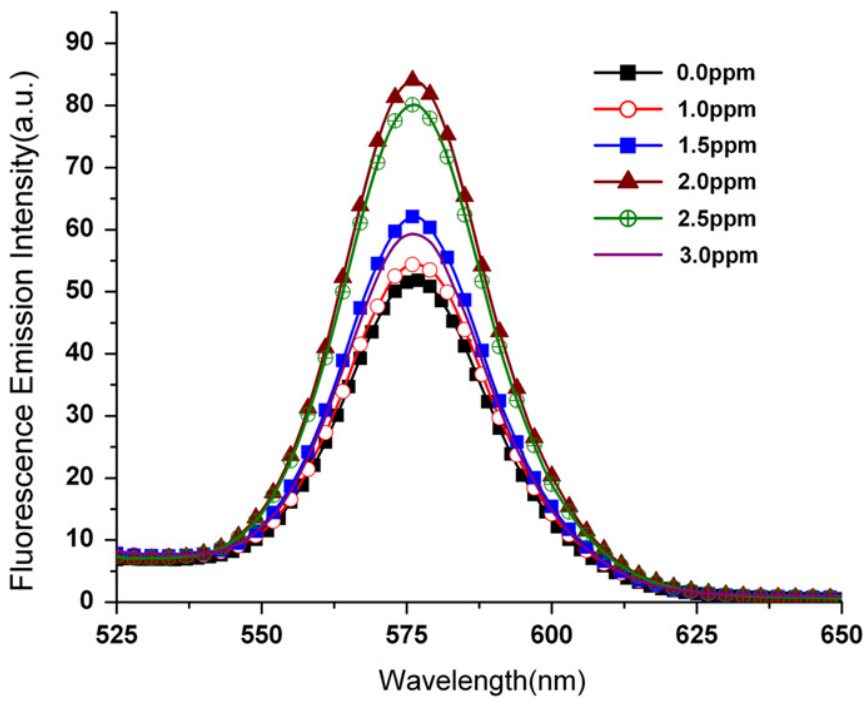

Fig. 6. Fluorescence emission spectra of the QD 570 quantum dots with varying Au NP concentration (0.0-4.0 ppm) in epoxy. The samples were excited at $500 \mathrm{~nm}$ and the emission collected at a $90^{\circ}$ angle to the excitation beam. (Note; the 3.5 and $4.0 \mathrm{ppm}$ data have not been shown in this figure to keep clarity. All data are shown in Fig. 7).

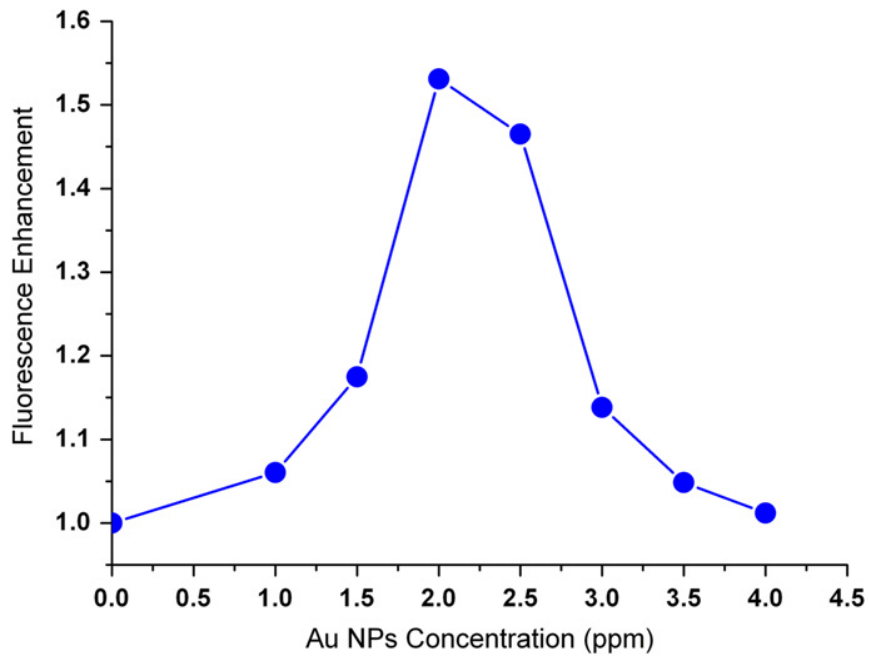

Fig. 7. Fluorescence enhancement as a function of Au NP concentration (ppm), relative to the total fluorescence of the $0.0 \mathrm{ppm}$ sample. The maximum enhancement was $53 \%$ for the $2.0 \mathrm{ppm}$ sample.

to fabricate the luminescent solar concentrator, as the overall optical efficiency mainly depends on the total emission of luminescent species.

\subsection{Fluorescence decay lifetime measurements}

The suggested origin of the decrease in emission intensity at high MNP concentration is confirmed by measurements of the emission lifetime of the QDs with and without the Au NPs. Samples $0.0 \mathrm{ppm}, 1.0 \mathrm{ppm}$, and $2.0 \mathrm{ppm}$ exhibit single exponential lifetime decays. In contrast, samples with $3.0 \mathrm{ppm}$ and $4.0 \mathrm{ppm}$ show double exponential decays as summarized in Table 1 and Fig. 8.

In general, emission quenching and decay lifetime shortening are the main evidence for the occurrence of non-radiative energy transfer in the optical emitter. Our observations support these two characteristics in QD/Au NP composites at higher MNP concentrations. The emission lifetime measurements support the existence of distinctive states for CdSe/ZnS QDs at higher Au NP 
concentrations in the composite samples shown in Table 1. As the concentration of Au NPs increases a subset of excited state QDs transfer the excited electron and hole to the Au NPs through nonradiative relaxation. The double exponential decay lifetime profile confirms two distinctive subsets of similar QDs, corresponding to the fast and to the slow decay components, $\tau_{1}$ and $\tau_{2}$, respectively. The slow component corresponds to radiative decay of the QDs while the fast component corresponds to those QDs that transfer their electron and hole non-radiatively to Au NPs.

\subsection{Enhanced excitation rate of $Q D s$}

We assign the initial increase of the emission with increasing Au NP concentration to an increased excitation of the QDs. This phenomenon is more pronounced when the SPR wavelengths of the Au NPs coincide with the absorption band of the QDs (in this case the SPR wavelength of the Au NPs and QD absorption band are around $560 \mathrm{~nm}$ ). The enhanced absorption (Fig. 5) and enhanced local electric field intensity at the SPR wavelength lead to an increased excitation rate of QDs, which is directly proportional to the square of the electric field amplitude [32].

We have proposed that the fluorescence emission intensity is increased due to the modified excitation rate of the QDs in the presence of Au NPs. To support this proposition we have carried out an excitation wavelength dependent study of the $2.0 \mathrm{ppm}$

Table 1

Fluorescence lifetime decay parameters (lifetimes ${ }^{\mathrm{a}}$, fractions, average lifetime ${ }^{\mathrm{b}}$ and fitting parameter ${ }^{\mathrm{C}}$ ) for $\mathrm{CdSe} / \mathrm{ZnS}$ quantum dots with and without Au NPs in epoxy. Samples 3.0 and $4.0 \mathrm{ppm}$ exhibit double exponential decay.

\begin{tabular}{lrlllll}
\hline Samples & $\tau_{2}(\mathrm{~ns})$ & $\tau_{2}(\mathrm{~ns})$ & $A_{1}$ & $A_{2}$ & $\tau_{\mathrm{av}}(\mathrm{ns})$ & $\chi^{2}$ \\
\hline $0.0 \mathrm{ppm}$ & 13.28 & & & & 13.28 & 1.010 \\
$1.0 \mathrm{ppm}$ & 12.49 & & & & 12.49 & 1.035 \\
$2.0 \mathrm{ppm}$ & 12.04 & & & & 12.04 & 1.040 \\
$3.0 \mathrm{ppm}$ & 11.66 & & & & 11.66 & 1.149 \\
$3.0 \mathrm{ppm}$ & 6.30 & 20.87 & 159.79 & 113.64 & 10.35 & 1.039 \\
$4.0 \mathrm{ppm}$ & 9.39 & & & & 9.39 & 1.30 \\
$4.0 \mathrm{ppm}$ & 5.38 & 17.50 & 189.97 & 115.63 & 9.96 & 1.042
\end{tabular}

a Intensity decay equation; $I(t)=A_{1} \exp \left(-t / \tau_{1}\right)+A_{2} \exp \left(-t / \tau_{2}\right)$. The decay parameters $\tau_{1}, \tau_{2}, A_{1}$, and $A_{2}$ are the lifetimes and their corresponding amplitudes.

${ }^{\mathrm{b}}$ Average decay lifetime, $\tau_{\mathrm{av}}=\left(\tau_{1} A_{1}+\tau_{2} A_{2}\right) /\left(A_{1}+A_{2}\right)$.

c Goodness of fit parameter, $\chi^{2}=1$ for a good fit [44].

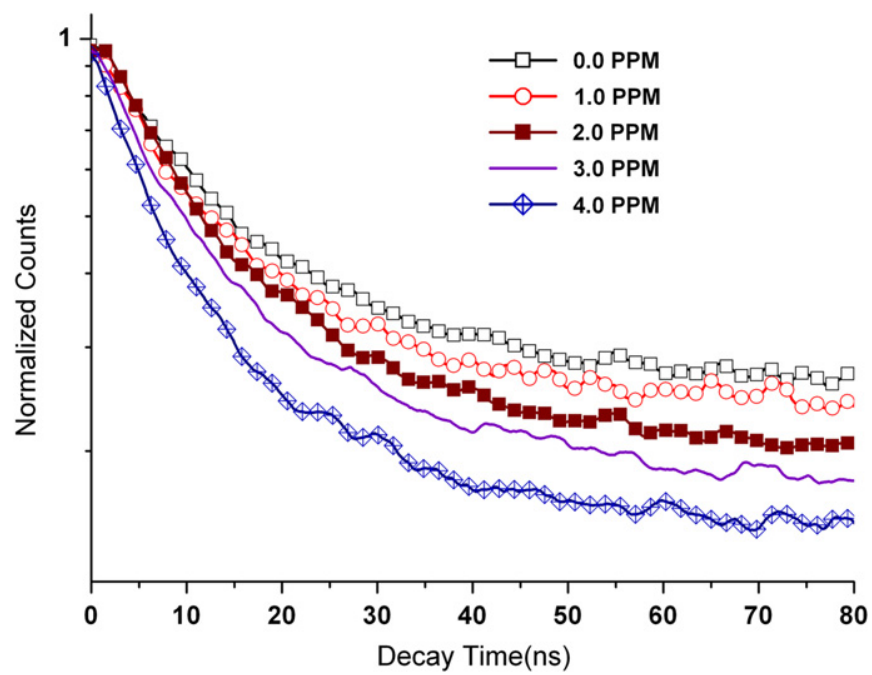

Fig. 8. Normalized fluorescence lifetime decay profile for the QD 570 quantum dots with varying Au NP concentrations (0.0-4.0 ppm) in epoxy. (Note; the 1.5 2.5 , and $3.5 \mathrm{ppm}$ data have not been shown in this figure to keep clarity in figure). sample. Fluorescence emission was collected as the sample was excited at wavelengths in the range $400-525 \mathrm{~nm}$. (Note: the SPR wavelength for Au NPs is at $560 \mathrm{~nm}$ ). Fig. 9 shows the variation in emission intensity as a function of excitation wavelength. A QD near Au NPs interacts with both the incident and the plasmonenhanced field. However, the coupling of the plasmon-enhanced field to the QD can become much larger than the incident field when the dipole frequency is in near resonance with the Au NP surface plasmon. The amplitude of the surface plasmon-induced electromagnetic field in Au NPs is expected to be much greater than that of the incident electromagnetic field at the SPR wavelength [26,42]. Consequently (Fig. 9.), the overall fluorescence emission for the $2.0 \mathrm{ppm}$ sample increases as the excitation wavelength approaches the SPR wavelength of the Au NP.

However, off-resonance, the total enhancement factor is still greater than 1.0. This could be explained by increased absorption and enhanced emission rate of the QDs. It is known that emission enhancement occurs when the MNP surface plasmon resonance wavelength coincides with the emitter emission band [36-38]. Here, the broad SPR wavelength of the Au NPs has some overlap with the emission band of the QDs as shown in Fig. 10. This implies the possibility of an increase in emission rate and consequently, increased emission intensity that may explain these results at off-resonance wavelengths.

\section{Conclusions}

We have studied the influence of the plasmon-induced electromagnetic field of Au NPs on the fluorescence properties of core-shell type $\mathrm{CdSe} / \mathrm{ZnS}$ quantum dots in an epoxy, which is appropriate for fabrication of quantum dot solar concentrators (QDSCs). The QD concentration used was $0.008 \%$ (weight percentage) and the Au NP concentration varied from 1.0 to $4.0 \mathrm{ppm}$ in the epoxy resin.

Optical absorption increased with increasing Au NP concentration and this is attributed to enhanced local photon mode density of incident light in the vicinity of QDs. Fluorescence emission is highly dependent on the concentration of Au NPs. As the concentration increases the emission intensity exhibits, first enhancement, and then diminishment. A maximum emission enhancement of $53 \%$ was measured for the composite with a $2.0 \mathrm{ppm}$ concentration of Au NPs. The enhancement of QD emission is mainly due to increased QD excitation rates, and the results suggest there may

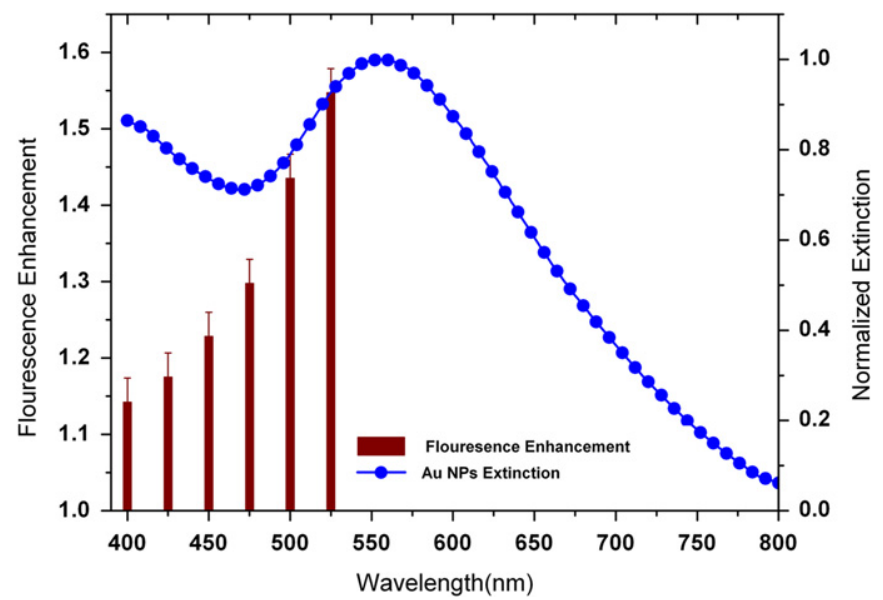

Fig. 9. Fluorescence enhancement as a function of excitation wavelength for the $2.0 \mathrm{ppm}$ sample. Excitation enhancement was obtained by taking the ratio of the total emission of the $2.0 \mathrm{ppm}$ sample to that of the $0.0 \mathrm{ppm}$ sample at the particular excitation wavelength (solid bar). The normalized extinction spectra (solid circles) of the Au NPs are also plotted to illustrate the origin of the effect. 


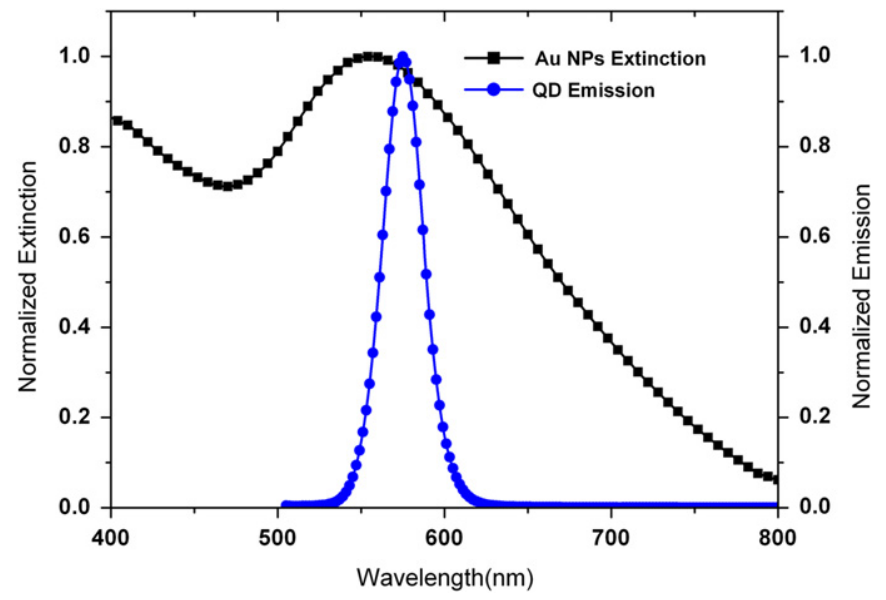

Fig. 10. Au NP extinction (squares) and QD emission (solid circles) spectra; illustrating the slight overlap of the QD emission and the SPR wavelength of the Au NPs.

also be an increase in the emission rate of the QDs due to the surface plasmon resonance enhanced local electric field of the $\mathrm{Au}$ NPs. Higher concentrations of Au NPs led to decreases in fluorescence intensity, which we interpret as being due to non-radiative transfer of the excited carriers from the QDs to nearby Au NPs. This interpretation is supported by a measured decrease in fluorescence decay lifetime of the QDs and the emergence of double exponential decay profiles for higher Au NP concentrations. The increased excitation rate of the QDs due to the surface plasmon resonance has been verified by an excitation wavelength dependent study of the $2.0 \mathrm{ppm}$ sample. The fluorescence enhancement increases as the excitation wavelength approaches the SPR wavelength of Au NPs. In summary plasmonic interaction has been shown to increase the fluorescence emission of QDs. By exploiting this plasmonic interaction, higher efficiency QDSCs may be developed.

\section{Acknowledgments}

This research work is funded by Higher Education Authority (HEA), Ireland, under the Postgraduate R \& D skills program.

\section{References}

[1] A. Goetzberger, W. Greubel, Solar energy conversion with fluorescent collectors, Journal of Applied Physics 14 (1977) 123-139.

[2] T. Trupke, M.A. Green, P. Wurfel, Improving solar cell efficiencies by downconversion of high energy photons, Journal of Applied Physics 92 (2002) 1668-1674.

[3] A. Goetzberger, Fluorescent solar collector: operating condition with diffuse light, Journal of Applied Physics 16 (1978) 399-404.

[4] A. Goetzberger, V. Wittwer, Fluorescent planar collector concentrators for solar energy conversion, Festkorperprobleme 19 (1979) 427-450.

[5] W.H. Weber, J. Lambe, Luminescent greenhouse collector for solar radiation, Applied Optics 15 (1976) 2299-2300.

[6] V. Witter, Theory of fluorescent planer concentrator and experimental result Journal of Luminescence 24-25 (1981) 873-876.

[7] C.F. Rapp, N.L. Boling, Luminescent solar concentrator, Proceedings of the Thirteenth IEEE Photovoltaic Specialists Conference, 1978, pp. 690-693.

[8] K. Barnham, J.L. Marques, J. Hassard, P. O'Brien, Quantum dot concentrator and thermodynamic model for the global red-shift, Applied Physics Letters 76 (2000) 1197-1199.

[9] A.P. Alivisatos, Perspective on the physical chemistry of semiconductor nanocrystals, Journal of Physical Chemistry 100 (1996) 13226-13239.

[10] A.P. Alivisatos, Electrical Studies of semiconductor nanocrystal colloids, MRS Bulletin 23 (1998) 18-22.

[11] H. Du, C. Chen, R. Krishnan, T.D. Krauss, J.M. Harbold, F.W. Wise, M.G. Thomas, J. Silcox, Optical properties of colloidal PbSe nanocrystal, Nano Letters 2 (2002) 1321-1324.

[12] S.J. Gallagher, B. Norton, P.C. Eames, Quantum dot solar concentrators: electrical conversion efficiencies and comparative concentrating factors of fabricated devices, Solar Energy 81 (2007) 813-821.
[13] V. Sholin, J.D. Olson, S.A. Carter, Semiconducting polymers and quantum dots in luminescent solar concentrator for solar energy harvesting, Journal of Applied Physics 101 (2007) 123114-123122.

[14] M.G. Hyldahl, S.T. Bailey, B.P. Wittmershaus, Photo-stability and performance of CdSe/ZnS quantum dots in luminescent solar concentrators, Solar Energy 83 (2009) 566-573.

[15] J. Bomm, A. Buchtemann, A.J. Chatten, R. Bose, D.J. Farrell, N.L.A. Chan, Y. Xiao, L.H. Slooff, T. Meyer, A. Meyer, W.G.J.H.M. van Sark, R. Koole, Fabrication and full characterization of state-of-the-art quantum dot luminescent solar concentrators, Solar Energy Materials and Solar Cells 95 (2011) 2087-2094.

[16] Nanoco Technology, 〈http://www.nanocotechnologies.com/〉 (accessed 03.04.11).

[17] Evident Technology, 〈http://www.evidenttech.com/ > (accessed 05.07.11).

[18] B.C. Rowan, L.R. Wilson, B.S. Richards, Advanced material concepts for luminescent solar concentrator, IEEE Journal of Selected Topics in Quantum Electronics 14 (2008) 1312-1322.

[19] A.J. Chatten, K.W.J. Barnham, B.F. Buxton, N.J. Ekins-Daukes, and M.A. Malik, The quantum dot concentrator: theory and results, Proceedings of Third World Congress on Photovoltaic Energy Conversion (WPEC-3), Osaka, Japan, 2003, pp. 2657-2660.

[20] A.J. Chatten, K.W.J. Barnham, B.F. Buxton, N.J. Ekins-Daukes, M.A. Malik, Quantum dot solar concentrators, Semiconductors 38 (2004) 909-917.

[21] A.J. Chatten, K.W.J. Barnham, B.F. Buxton, N.J. Ekins-Daukes, M.A. Malik, New approach to modelling quantum dot concentrators, Solar Energy Materials and Solar Cells 75 (2003) 363-371.

[22] PlasmaChem GmbH, 〈http://www.plasmachem.com/〉 (accessed 01.07.11).

[23] P.J. Schuck, D.P. Fromm, A. Sundaramurthy, G.S. Kino, W.E. Moerner, Improving the mismatch between light and nanoscale objects with gold nanoantennas, Physical Review Letters 94 (2005) 017402-017405.

[24] N. Calander, M. Willander, Theory of surface-plasmon resonance optical field enhancement at prolate spheroids, Journal of Applied Physics 92 (2002) 4878-4884.

[25] P. Muhlschlegel, H.J. Eisler, O.J.F. Martin, B. Hecht, D.W. Pohl, Resonant optical antennas, Science 308 (2005) 1607-1609.

[26] E. Hao, G.C. Schatz, Electromagnetic fields around silver nanoparticles and dimers, Journal of Chemical Physics 120 (2004) 357-366.

[27] W.L. Barnes, A. Dereux, T.W. Ebbesen, Surface plasmon subwavelength optics, Nature 424 (2003) 824-830.

[28] E.M. Purcell, Spontaneous emission probabilities at radio frequencies, Physical Review 69 (1946) 681.

[29] K.H. Drexhage, Interaction of light with monomolecular dye layers, Progress in Optics 12 (1974) 163-232.

[30] D. Kleppner, Inhibited spontaneous emission, Physics Review Letters 47 (1981) 233-236.

[31] W.L. Barnes, Fluorescence near interfaces: the role of photonic mode density, Journal of Modern Optics 45 (1998) 661-699.

[32] P. Anger, P. Bharadwaj, L. Novotny, Enhancement and quenching of singlemolecule fluorescence, Physical Review Letters 96 (2006) 113002-113005.

[33] Y. Chen, K. Munechika, I.J. Plante, A.M. Munro, S.E. Skrabalak, Y. Xia D.S. Ginger, Excitation enhancement of CdSe quantum dots by single metal nanoparticles, Applied Physics Letters 93 (2008) 053106-053108.

[34] O. Kulakovich, N. Strekal, A. Yaroshevich, S. Maskevich, Enhanced luminescence of CdSe quantum dots on Gold colloids, Nano Letters 2 (2002) 1449-1452.

[35] O. Stranik, H.M. McEvoy, C. McDonagh, B.D. MacCraith, Plasmonic enhancement of fluorescence for sensor applications, Sensors and Actuators B 107 (2005) 148-153.

[36] Y. Chen, K. Munechika, D.S. Ginger, Dependence of fluorescence intensity on the spectral overlap between fluorophores and plasmon resonant single silver nanoparticles, Nano Letters 7 (2007) 690-696.

[37] J.S. Biteen, D. Pacifici, N.S. Lewis, H.A. Atwater, Enhanced radiative emission rate and quantum efficiency in coupled silicon nanocrystal-nanostructured Gold emitters, Nano Letters 5 (2005) 1768-1773.

[38] J.S. Biteen, N.S. Lewis, H. Mertens, A. Polman, H.A. Atwater, Spectral tuning of plasmon-enhanced silicon quantum dot luminescence, Applied Physics Letters 88 (2006) 131109-131111.

[39] S. Chandra, S.J. McCormack, J. Doran, M. Kennedy, A.J. Chatten, New concept for luminescent solar concentrator, 25th European Photovoltaic Solar Energy Conference, 2010, Valencia, Spain, pp. 759-762.

[40] G. Xua, Y. Chen, M. Tazawab, P. Jin, Influence of dielectric properties of substrate upon plasmon resonance spectrum of supported Ag nanoparticles, Applied Physics Letters 88 (2006) 043114-043116.

[41] S.J. Gallagher, B.C. Rowan, J. Doran, B. Norton, Quantum dot solar concentrator: device optimization using spectroscopic techniques, Solar Energy 81 (2007) 540-547.

[42] J.P. Kottmann, O.J.F. Martin, D.R. Smith, S. Schultz, Dramatic localized electromagnetic enhancement in plasmon resonant nanowire, Chemical Physics Letters 341 (2001) 1-6.

[43] F. Stietz, J. Bosbach, T. Wenzel, T. Vartanyan, A. Goldmann, F. Trager, Decay times of surface plasmon excitation in metal nanoparticles by persistent spectral hole burning, Physical Review Letters 84 (2000) 5644-5647.

[44] D.V. O'Connor, D. Phillips, Time Correlated Single Photon Counting, Academic Press, NY, USA, 1984 